\title{
Local Conformal Field Algebras
}

\author{
Denis Juriev \\ TFFA, Department of Mathematics, Moscow State University, SU-119899 Moscow, USSR \\ Received October 31, 1990
}

\begin{abstract}
The local conformal field algebras with the multiplication corresponding to the regularized pointwise product of fields in the operator algebras of the quantum conformal field theory are investigated.
\end{abstract}

\section{Introduction}

The scenery of the theory of groups, their representations and homogeneous spaces in view of the intensive interaction with mathematical physics deeply depends on changes in it. Thus, forming the spectrum of problems of modern quantum field theory (string field theory, conformal field theory, etc.) provoked the elaboration of a "daughter" rapidly developping discipline including infinite dimensional geometry, harmonic analysis on the infinite dimensional manifolds and the theory of representations of the infinite dimensional algebras and groups.

At the same time methods of quantum field theory penetrated into abstract mathematics (topological quantum field theory [1-4]). There exist some arguments for the hypothesis that quantum conformal field theory [5-8] would be useful in singularity theory. One should suppose that the operator formalism of quantum field theory would play the role analogous to that of cohomology theory in topology.

All these circumstances explain the appearance of this paper devoted to the algebraic aspects of QCFT as the choice of the approach to this theory based on infinite dimensional geometry [9-20]. We construct the $L$-algebra $L(C$ vir), whose elements belong to the Fock space over the universal deformation of the complex disc, in which the model of the Verma modules over the Virasoro algebra is realised [20]. Then we investigate the local conformal field algebras (LCFAs), which may be characterised as algebras of geometric objects on the complex plane valued in the $L$-algebra $L(C$ vir). The multiplication in the LCFAs corresponds to the regularized pointwise product of fields in the operator algebras of QCFT.

For pedagogical purposes we shall consider the simpler case of the $s l(2, C)$ invariant field theory before the conformal one throughout the text. 


\section{The Model of the Verma Modules over the Virasoro Algebra}

As stated in the introduction we shall consider the simpler case of the Lie algebra $\operatorname{sl}(2, C)$ before the Virasoro one.

Definition 1a [20]. The model of the Verma modules over $\operatorname{sl}(2, C)$ is the representation of this algebra in the direct integral

$$
\oint V_{h} d h
$$

of the Verma modules [21] over $\operatorname{sl}(2, C)$.

Definition 1b [20]. The model of the Verma modules over the Virasoro algebra $C$ vir is the representation of this algebra in the direct integral

$$
\oint V_{h, c} \dot{d h}
$$

of the Verma modules over $C$ vir $[19,22]$ with fixed central charge $c$.

In [20] the realisations of the models of the Verma modules over $C$ vir and $\operatorname{sl}(2, C)$ in the Fock spaces over the deformations of the complex disc were constructed. The deformation corresponding to $\operatorname{sl}(2, C)$ determines the Laguerre realisation of the Lobachevskii geometry. The model space consists of all holomorphic functions of two variables $z$ and $w$, where $z$ belongs to the complex plane $C$ and $w$ belongs to the universal covering $\tilde{C}^{*}$ of the complex plane without the initial point $C^{*}=C \backslash 0$. The action of $\operatorname{sl}(2, C)$-generators is determined by the next formulae:

$$
\begin{aligned}
L_{-1} & =z \\
L_{0} & =z \frac{\partial}{\partial z}-w \frac{\partial}{\partial w}, \\
L_{1} & =z\left(\frac{\partial}{\partial z}\right)^{2}-2 w \frac{\partial^{2}}{\partial w \partial z}+w^{2} \frac{\partial}{\partial w} .
\end{aligned}
$$

Proposition 1. The highest vectors in the model of the Verma modules over sl $(2, C)$ defined by (1) have the form

$$
v_{h}=w^{-h} F(-h, 2-2 h ; z w)
$$

where $F(a, b ; u)$ is the degenerate hypergeometric function [23].

Proof. To find the highest vector $v_{h}$ with the highest weight $h$ in the model means to find the function $f_{h}(w, z)$ of two complex variables $z$ and $w$, which obeys the system of differential equations

$$
\begin{array}{r}
\left(z \frac{\partial}{\partial z}-w \frac{\partial}{\partial w}-h\right) f_{h}(w, z)=0 \\
\left(z\left(\frac{\partial}{\partial z}\right)^{2}-2 w \frac{\partial^{2}}{\partial w \partial z}+w^{2} \frac{\partial}{\partial w}\right) f_{h}(w, z)=0 .
\end{array}
$$

The general solution $f_{h}(w, z)$ of the first equation may be represented as

$$
f_{h}(w, z)=w^{-h} F_{h}(z w) .
$$


Substituting this expression for $f_{h}$ in the second equation one will obtain the next equation for $F_{h}$,

$$
F_{h}^{\prime \prime}(u)+(2-2 h-u) F_{h}^{\prime}(u)+h F_{h}(u)=0,
$$

which coincides with the degenerate hypergeometric equation

$$
y^{\prime \prime}+(b-u) y^{\prime}-a y=0
$$

with the parameters $a$ and $b$ equal to $-h$ and $2-2 h$, respectively.

The comparison of formulae (2), (4), (5) finishes the proof.

The model of the Verma modules over the Virasoro algebra may be realised in the Fock space over the universal deformation of the complex disc [20]. The model space consists of all holomorphic functions of variables $w, c_{1}, c_{2}$, $c_{3}, \ldots c_{n}, \ldots$, where $c_{k}$ belongs to the complex plane $C$ for all $k$ and $w$ belongs to the universal covering $\tilde{C}^{*}$ of the complex plane without the initial point $C^{*}=C \backslash 0$. The action of $C$ vir is defined by the formulae

$$
\begin{aligned}
L_{-p}= & \sum_{k \geq 1}(k+1) c_{k+p} \frac{\partial}{\partial c_{k}}+c_{p}, \quad p<0, \\
L_{0}= & \sum_{k \geq 1} k c_{k} \frac{\partial}{\partial c_{k}}-w \frac{\partial}{\partial w}, \\
L_{1}= & \sum_{k \geq 1} c_{k}\left((k+2) \frac{\partial}{\partial c_{k+1}}-2 \frac{\partial^{2}}{\partial c_{1} \partial c_{k}}\right)-2 w \frac{\partial^{2}}{\partial w \partial c_{1}}+w^{2} \frac{\partial}{\partial w}, \\
L_{2}= & \sum_{k \geq 1} c_{k}\left((k+3) \frac{\partial}{\partial c_{k+2}}-\left(4 \frac{\partial}{\partial c_{2}}-\left(\frac{\partial}{\partial c_{1}}\right)^{2}\right) \frac{\partial}{\partial c_{k}}\right. \\
& \left.-b_{k}\left(\frac{\partial}{\partial c_{1}}, \ldots \frac{\partial}{\partial c_{k+2}}\right)\right)-\left(4 \frac{\partial}{\partial c_{2}}-\left(\frac{\partial}{\partial c_{1}}\right)^{2}\right) w \frac{\partial}{\partial w} \\
& +3 w^{2} \frac{\partial^{2}}{\partial w \partial c_{1}}-w^{3} \frac{\partial}{\partial w}+0.5 c\left(\frac{\partial}{\partial c_{2}}-\left(\frac{\partial}{\partial c_{1}}\right)^{2}\right) \\
L_{n}= & \frac{(-1)^{n}}{(n-2) !}\left(\operatorname{ad}\left(L_{1}\right)\right)^{n-2} L_{2} ; \quad n>2
\end{aligned}
$$

where $b_{k}\left(u_{1}, \ldots u_{k+2}\right)$ are the Laurent coefficients of the function $1 /\left(w^{2}\left(1+u_{1} w+\right.\right.$ $\left.\left.u_{2} w^{2}+\ldots\right)\right)$.

Proposition 2. The highest vectors in the model of the Verma modules over the Virasoro algebra defined by (6) have the form

$$
v_{h}=w^{-h} G\left(c ; h ; c_{1} t, c_{2} t^{2}, \ldots c_{n} t^{n}, \ldots\right)
$$


where the function $G\left(c ; h ; u_{1}, u_{2}, u_{3}, \ldots u_{n}, \ldots\right)$ obeys the system of differential equations

$$
\begin{aligned}
& \sum_{k \geq 1} u_{k}\left((k+2) \frac{\partial}{\partial u_{k+1}}-2(k+1) \frac{\partial^{2}}{\partial u_{1} \partial u_{k}}-k \frac{\partial}{\partial u_{k}}\right) G \\
& -2(1-h) \frac{\partial}{\partial u_{1}} G-h G=0 \\
& \sum_{k \geq 1} u_{k}\left((k+3) \frac{\partial}{\partial u_{k+2}}-(k+1)\left(4 \frac{\partial}{\partial u_{2}}-\left(\frac{\partial}{\partial u_{1}}\right)^{2}\right) \frac{\partial}{\partial u_{k}}\right. \\
& \left.\left.-b_{k}\left(\frac{\partial}{\partial u_{1}}, \ldots \frac{\partial}{\partial u_{k+2}}\right)\right)+3 k \frac{\partial^{2}}{\partial u_{1} \partial u_{k}}-k \frac{\partial}{\partial u_{k}}\right) G \\
& -(2-h)\left(4 \frac{\partial}{\partial u_{2}}-\left(\frac{\partial}{\partial u_{1}}\right)^{2}\right) G-3(1-h) \frac{\partial}{\partial u_{1}} g+h G \\
& +0.5 c\left(\frac{\partial}{\partial u_{2}}-\left(\frac{\partial}{\partial u_{1}}\right)^{2}\right) G=0 .
\end{aligned}
$$

Proof. To find the highest vector $v_{h}$ with the highest weight $h$ in the model space means to find the function $g_{h, c}\left(w, c_{1}, c_{2}, \ldots c_{n}, \ldots\right)$, which obeys the closed system of the differential equations

$$
\begin{aligned}
\left(L_{0}-h\right) g_{h, c}\left(w, c_{1}, c_{2}, \ldots c_{n}, \ldots\right) & =0, \\
L_{k} g_{h, c}\left(w, c_{1}, c_{2}, \ldots c_{n}, \ldots\right) & =0, \quad k>0
\end{aligned}
$$

The general solution $g_{h, c}\left(w, c_{1}, \ldots c_{n}, \ldots\right)$ of the first equation may be represented as

$$
g_{h, c}\left(w, c_{1}, c_{2}, \ldots c_{n}, \ldots\right)=t^{-h} G_{h, c}\left(c_{1} t, \ldots c_{n} t^{n}, \ldots\right) .
$$

Mentioning that the equations of the second group in (9) are the sequences of the first two of them and substituting the expression for $g_{h, c}(10)$ in these two equations, one will obtain the system of equations (8).

It can be shown that the function $G\left(c ; h ; u_{1}, u_{2}, \ldots u_{k}, \ldots\right)$ is a special function of hypergeometric type (the degenerate hypergeometric function, corresponding to the bibundle of the universal deformation of the complex disc over the flag manifold of the Virasoro-Bott group [16-19] and the complex disc [24]).

\section{The $L$-Algebra $L\left({ }_{b} i C\right.$ vir $)$}

Definition 2. Let $R$ be an associative algebra with the unit over the field $K, g-$ the Lie subalgebra of $\operatorname{Der}(R)$. Then an associative algebra $A$ with the unit over the field $K$ will be called an $L$-algebra over the pair $(r, g)$ iff $A$ is the right $R$ module (so $R$-bimodule in a natural way) and the $g$-module so that the structure of the $g$-module is compatible with the structure of the left $R$-module.

If $R$ is the commutative algebra of holomorphic functions on a complex manifold $X$ and $g$ is an algebra of vector fields on $X$ then $A$ is the $L$-algebra over $(R, g)$ iff $A$ is the local associative algebra on $X$ (the right $R$-module) with the unit over the complex numbers and also

$$
[L(\xi), T(\varphi)]=T(\xi \cdot \varphi),
$$


where $\varphi \in r, \xi \in g, T$ is the operator of the left multiplication in $A, L \in$ $\operatorname{Hom}(g, \operatorname{End}(A))$.

Let 0 be the commutative algebra of all holomorphic functions on $\tilde{C}^{*}$, namely, $0=\left\{\int F(x) w^{x} d x\right\}$. Then the models (1) and (6) are the right 0 -modules in a natural way.

Proposition 2a. The model (1) of the Verma modules over the Lie algebra $\operatorname{sl}(2, C)$ possesses exactly one structure of the L-algebra over $(0, s l(2, C))$ compatible with the structure of the right 0 -module and sl $(2, C)$-module in the model (1) so that

$$
L_{-1} T(\Phi)=T\left(L_{-1} \Phi\right)
$$

for all $\Phi$ from the model space.

Proof. We shall present this algebra below. Its uniqueness follows from the fact that the model as the $\operatorname{sl}(2, C)$-module is generated by the linear subspace 0 in it. This statement is explicated in the following lemma.

Lemma 1. Let $T$ be the operator of the left multiplication in an L-algebra over $(0, \operatorname{sl}(2, C))$ in the model $(1)$, then

$$
\begin{aligned}
T(z) & =L_{-1}, \\
T(w) & =w-\frac{\partial}{\partial z} .
\end{aligned}
$$

Proof. The expression for the operator $T(z)$ is evidently obtained from (12). Let's find the expression for $T(w)$. The condition

$$
\left[L_{-1}, T(w)\right]=I
$$

allows us to choose the following ansatz for $T(w)$ :

$$
T(w)=A\left(w, z, \frac{\partial}{\partial w}\right)-\frac{\partial}{\partial z} .
$$

To find the form of the operator $A\left(w, z, \frac{\partial}{\partial w}\right)$ one should use the next property of $T(w)$,

$$
T(w) \varphi(w)=w \varphi(w),
$$

and obtain that $A\left(w, z, \frac{\partial}{\partial w}\right)=w$.

Corollary.

$$
\begin{aligned}
T\left(w^{h}\right)= & w^{h}\left(1-w^{-1} \frac{\partial}{\partial z}\right)^{h}=w^{h}-h w^{h-1} \frac{\partial}{\partial z} \\
& +\frac{h(h-1)}{2} w^{h-2}\left(\frac{\partial}{\partial z}\right)^{2}-\frac{h(h-1)(h-2)}{6} w^{h-3}\left(\frac{\partial}{\partial z}\right)^{3}+\ldots
\end{aligned}
$$

Theorem 1a. The correspondence

$$
z^{k} w^{h}\left(\frac{\partial}{\partial t}\right)^{k} t^{h}
$$


introduces the structure of the L-algebra over $(0, s l(2, C))$ in the model. We shall denote the obtained L-algebra as $L(\operatorname{sl}(2, C))$. The statement above means that the L-algebra $L(\operatorname{sl}(2, C))$ as an associative algebra is isomorphic to the algebra of all differential operators of the variable $w$.

Proof. To prove the theorem we should check the following relations:

and

$$
\left[L_{0}, T(w)\right]=-T(w)
$$

$$
\left[L_{1}, T(w)\right]=(T(w))^{2} .
$$

Namely,

$$
\begin{aligned}
{\left[L_{0}, T(w)\right] } & =\left[z \frac{\partial}{\partial z}-w \frac{\partial}{\partial w}, w-\frac{\partial}{\partial z}\right] \\
& =-\left(w-\frac{\partial}{\partial z}\right)=-T(w) \\
{\left[L_{1}, T(w)\right] } & =\left[z\left(\frac{\partial}{\partial z}\right)^{2}-2 w \frac{\partial^{2}}{\partial w \partial z}+w^{2} \frac{\partial}{\partial w}, w-\frac{\partial}{\partial z}\right] \\
& =\left(\frac{\partial}{\partial z}\right)^{2}-2 w \frac{\partial}{\partial z}+w^{2}=\left(w-\frac{\partial}{\partial z}\right)^{2}=(T(w))^{2} \text {. Q.E.D. }
\end{aligned}
$$

Let's now consider the Virasoro case.

Proposition 2b. The model (6) of the Verma modules over the algebra $C$ vir possesses exactly one structure of the L-algebra over $\left(0, C \operatorname{vir}_{\mathrm{reg}}\right)\left(C \operatorname{vir}_{\mathrm{reg}}:=\operatorname{span}\left(L_{p}\right.\right.$, $p=-1,0,1,2,3,4, \ldots)$ ) compatible with the structure of the right 0 -module and

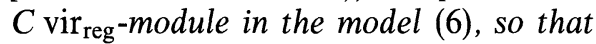

$$
L_{p} T(\Phi)=T\left(L_{p} \Phi\right), \quad P=-1,-2,-3, \ldots
$$

for all $\Phi$ from the model space.

Proof. We shall present this algebra below. Its uniqueness follows from the next lemma.

Lemma 2. Let $T$ be the operator of the left multiplication in an L-algebra over $\left(0, C\right.$ vir $\left._{\mathrm{reg}}\right)$ in the model (6) then

$$
\begin{aligned}
T\left(c_{p}\right) & =L_{-p}, \\
T(w) c_{1} & =c_{1} w-1, \\
T(w) c_{2} & =c_{2} w+w^{-1} .
\end{aligned}
$$

Proof. The expression for the operator $T\left(c_{p}\right)$ is evidently obtained from (16). The proof of the identity $(17 \mathrm{~b})$ is analogous to one from Lemma 1 . Let's prove $\left[L_{0}, T(w)\right]=-T(w)$ that $T(w) c_{2}$ may be represented as

$$
T(w) c_{2}=\sum_{k=0}^{N} w^{k-1} R_{k}\left(c_{1}, \ldots c_{n}, \ldots\right),
$$

where $\operatorname{deg}\left(R_{k}\right)=k$ if $\operatorname{deg}\left(c_{k}\right)=k$. Using the property $\left[L_{1}, T(w)\right]=(T(w))^{2}$ and (17b) one should obtain that $N \leq 2$. The equality (17c) follows from this fact and the identities $\left[L_{2}, T(w)\right]=-(T(w))^{3}$ and $\left[L_{1}, T(w)\right]=(T(w))^{2}$. 
Lemma 3. Let $T$ be the operator of the left multiplication in the L-algebra $\left(0, C\right.$ vir $\left._{\text {reg }}\right)$ in the model (6). Then

$$
T(w)=\sum_{k=0}^{\infty}(-1)^{k} w^{1-k} P_{k}\left(\frac{\partial}{\partial c_{1}}, \ldots \frac{\partial}{\partial c_{k}}\right),
$$

where $t(w)=\sum w^{-1+k} P_{k}\left(c_{1}, \ldots c_{k}\right)$ is the covariantly constant section of $R^{0} p_{*}$ $(\mathcal{O} A / M)$ with respect to Gauss-Manin connection, where $p: A \rightarrow M$ is the projection of the universal deformation of the complex disc on the flag manifold of the Virasoro-Bott group [20].

Proof. It follows from the property $\left[L_{0}, T(w)\right]=-T(w)$ that the operator $T(w)$ may be represented as

$$
T(w)=\sum_{k=-N}^{\infty} w^{1-k} Q_{k}\left(c_{1}, \ldots c_{n}, \ldots \frac{\partial}{\partial c_{1}}, \ldots \frac{\partial}{\partial c_{n}}, \ldots\right),
$$

where $\operatorname{deg}\left(Q_{k}\right)=-k$. Using the identities $\left[L_{k}, T(w)\right]=(-T(w))^{1-k}$ one should obtain that $T(w)$ doesn't depend on the variables $c_{1}, \ldots c_{n}, \ldots$. Hence

$$
T(w)=\sum_{k=0}^{\infty} w^{1-k} Q_{k}\left(\frac{\partial}{\partial c_{1}}, \ldots \frac{\partial}{\partial c_{k}}\right) .
$$

Let's rewrite the identities $\left[L_{k}, T(w)\right]=(-T(w))^{1+k}$, transiting to the conjugate variables for $c_{1}, \ldots c_{n}, \ldots$. Then they should have the form $L_{k} t(w)=(t(w))^{1+k}$, where $L_{k}$ acts in $R^{0} p_{*}(\mathcal{O} A / M)[9-13,20]$. So $t(w)$ is the covariant transport of the function $w^{-1} \in \mathcal{O}\left(p^{-1}(0)\right)$ with respect to Gauss-Manin connection.

Remark.

$$
\begin{aligned}
& P_{0}=0 \\
& P_{1}\left(u_{1}\right)=u_{1} \\
& P_{2}\left(u_{1}, u_{2}\right)=u_{2}-u_{1}^{2} \\
& P_{3}\left(u_{1}, u_{2}, u_{3}\right)=u_{3}-3 u_{1} u_{2}+2 u_{1}^{3} \\
& P_{4}\left(u_{1}, u_{2}, u_{3}, u_{4}\right)=u_{4}-4 u_{1} u_{3}+10 u_{1}^{2} u_{2}-5 u_{1}^{4}-2 u_{2}^{2}, \\
& P_{k+1}=(2+k)^{-1}\left(2(1-k) u_{1} P_{k}+\sum_{k}\left((k+2) u_{k+1}-2 u_{1} u_{k}\right)\right. \\
& \left.\quad \times \frac{\partial}{\partial u_{k}} P_{k}-\sum_{\substack{i, j>1 \\
i+j=k+1}} P_{i} P_{j}\right) \cdot
\end{aligned}
$$

Question. What is the combinatorial meaning of the polynomials $P_{k}$ ?

Corollary.

$$
T\left(w^{h}\right)=w^{h}\left(1+\sum_{k>1} w^{-k} P_{k}\left(\frac{\partial}{\partial c_{1}}, \ldots \frac{\partial}{\partial c_{k}}\right)\right)^{h} .
$$

Corollary. The structure of the associative algebra, determined by the structure of the L-algebra $\left(0, C\right.$ vir $\left._{\text {reg }}\right)$ in the model (6), doesn't depend on the central charge $c$. 
Lemma 4. Let $T$ be the operator of the left multiplication in the L-algebra over $\left(0, C \operatorname{vir}_{\text {reg }}\right)$ in the model (6), then

$$
\begin{gathered}
{\left[T\left(c_{p}\right), T\left(c_{q}\right)\right]=(q-p) T\left(c_{p+q}\right),} \\
{\left[T\left(c_{p}\right), T(\varphi(w))\right]=T\left((-w)^{1-p} \varphi^{\prime}(w)\right) .}
\end{gathered}
$$

Proof. The identity (20a) is the trivial sequence of (17a). The identity (20b) follows from $(17 \mathrm{~b}),(17 \mathrm{c})$ and the associativity of the $L$-algebra over $\left(0, C\right.$ vir $\left._{\text {reg }}\right)$ in the model (6) when $p=1,2$. One should obtain the property (20b) from this fact and (20a).

Theorem 1b. The isomorphism between the associative algebra $U\left(L_{+}\right) \ltimes 0$, where $L_{+}=\operatorname{span}\left(L_{-p}, p>0\right)$ and the model (6) (both objects are considered as the vector spaces), defined as

$$
U\left(L_{+}\right) \ltimes 0 \ni L_{-k_{1}} \ldots L_{-k_{n}} w^{h} \rightarrow T\left(c_{k_{1}}\right) \ldots T\left(c_{k_{n}}\right) w^{h}
$$

introduces the structure of the L-algebra over $\left(0, C \mathrm{vir}_{\mathrm{reg}}\right)$ in the model (6). The obtained L-algebra will be denoted as $L(C$ vir).

Proof. One needs to verify that

$$
\begin{aligned}
& {\left[t\left(c_{2}\right), \sum_{k=0}^{\infty}(-1)^{k} w^{1-k} P_{k}\left(\frac{\partial}{\partial c_{1}}, \ldots \frac{\partial}{\partial c_{k}}\right)\right]} \\
& =\left(\sum_{k=0}^{\infty}(-1)^{k} w^{1-k} P_{k}\left(\frac{\partial}{\partial c_{1}}, \ldots \frac{\partial}{\partial c_{k}}\right)\right)^{-1} .
\end{aligned}
$$

This equality follows from the definition of the function $t(w)=\sum w^{-1+k}$ $\times P_{k}\left(c_{1}, \ldots c_{k}\right)$ as the covariant transport of the function $w^{-1} \in \mathcal{O}\left(p^{-1}(0)\right)$ with respect to Gauss-Manin connection, which is $C$ vir-invariant.

\section{Local Conformal Field Algebras (LCFAs)}

Definition 3. The $L$-algebra $B$ over $(R, g)$ will be called the $L^{o}$-algebra over $(R, g)$ iff $g \subset \operatorname{Der}(B)$. The $L^{o}$-algebra over $(0, C$ vir $)$ will be called the algebra of geometric objects on $\bar{C}^{*}$.

Definition 4. Let $A$ be the $L$-algebra over $(R, g)$, then the left $R$-module $V$ will be called the left module over the $L$-algebra $A$, iff $V$ is the left module over the associative algebra $A$ and the $g$-module so that these structures are compatible $\left(V\right.$ is the $\operatorname{alg}_{\operatorname{End}(A)}(U(g) T(A))$-module).

Definition 5. Let $A$ be the $L$-algebra over $(R, g)$ and $B$ the $L^{o}$-algebra over $(R, g)$. Then the $L$-algebra $C$ over $(R, g)$ will be called the local field algebra with the algebra of primary fields $B$ and the structural algebra $A$ iff

1. $C$ is the left $A$-module

2. $C$ is the $L$-algebra over $(B, g)$.

If $R=0, g=\operatorname{sl}(2, C), A=L(\operatorname{sl}(2, C))$ then $C$ will be called the local $s l(2, C)$-invariant field algebra or local projective field algebra (LPFA). If $R=0$, 
$\mathrm{g}=C \operatorname{vir}_{\mathrm{reg}}, A=L(C$ vir $)$ then $C$ will be called the local conformally invariant field algebra or local conformal field algebra (LCFA). The multiplication in the LPFAs and LCFAs may be considered as the pointwise regularisation of the operator product in operator algebras of quantum $s l(2, C)$-invariant and conformally invariant field theories.

Let's consider an arbitrary $L^{o}$-algebra $B$ over $(0, s l(2, C))$. The generators of the Lie algebra $\operatorname{sl}(2, C)$ in $B$ will be denoted as $l_{j}, j=-1,0,1$. The operator of the left multiplication in $B$ will be denoted as $\tau$. Let's consider the left module

$$
L(\operatorname{sl}(2, C) ; B)=L(\operatorname{sl}(2, C)) \otimes_{0} B
$$

this module should be identified with the space of all $B$-valued holomorphic functions of one complex variable $z$. Let's define the $s l(2, C)$-action in $L(s l(2, C) ; B)$ by the formulae

$$
\begin{aligned}
L_{-1} & =z, \\
L_{0} & =z \frac{\partial}{\partial z}+l_{0}, \\
L_{1} & =z\left(\frac{\partial}{\partial z}\right)^{2}+2 l_{0} \frac{\partial}{\partial z}+l_{1} .
\end{aligned}
$$

Lemma 5. $L(s l(2, C) ; B)$ is the left module over the L-algebra $L(s l(2, C))$.

Proof. One needs to verify

$$
\begin{aligned}
& {\left[L_{0}, T(t)\right]=-T(t),} \\
& {\left[L_{1}, T(t)\right]=(T(t))^{2} .}
\end{aligned}
$$

Namely,

$$
\begin{aligned}
{\left[L_{0}, T(t)\right] } & =\left[z \frac{\partial}{\partial z}+l_{0}, \tau(t)-\frac{\partial}{\partial z}\right] \\
& =\left[\frac{\partial}{\partial z}+\left[l_{0}, \tau(t)\right]\right]=\frac{\partial}{\partial z}-\tau(t)=-T(t), \\
{\left[L_{1}, T(t)\right] } & =\left[z\left(\frac{\partial}{\partial z}\right)^{2}+2 l_{0} \frac{\partial}{\partial z}+l_{1}, \tau(t)-\frac{\partial}{\partial z}\right] \\
& =\tau(t)^{2}-2 \tau(t) \frac{\partial}{\partial z}+\left(\frac{\partial}{\partial z}\right)^{2}=\left(\tau(t)-\frac{\partial}{\partial z}\right)^{2} \\
& =(T(t))^{2} .
\end{aligned}
$$

Proposition 3a. The left module $L(\operatorname{sl}(2, C) ; B)$ over the L-algebra $L(s l(2, C))$ possesses exactly one structure of the LPFA with the algebra of primary fields $B$.

Proof. We shall present this algebra below. Its uniqueness follows from the next lemma. 
Lemma 6. Let $T$ be the operator of the left multiplication in a LPFA, realised in $L(\operatorname{sl}(2, C) ; B)$, then

$$
T(\xi)=\tau(\xi)-\tau\left(l_{-1}(\xi)\right) \frac{\partial}{\partial z}+\tau\left(l_{-1}^{2}(\xi)\right) \frac{\left(\frac{\partial}{\partial z}\right)^{2}}{2}+\tau\left(l_{-1}^{3}(\xi)\right) \frac{\left(\frac{\partial}{\partial z}\right)^{3}}{6}+\ldots
$$

for all $\xi$ from $B$.

Proof. The statement of the lemma follows from the next equality,

$$
T(\xi) z=z \xi+l_{-1}(\xi)
$$

The equality (23) should be proved in the following manner:

$$
\begin{aligned}
T(\xi) z & =T(\xi) L_{-1} \mathbb{1}=L_{-1} T(\xi) \mathbb{1}-\left[L_{-1}, T(\xi)\right] \mathbb{1} \\
& =z \xi-T\left(l_{-1}(\xi)\right) \mathbb{1}=z \xi-l_{-1}(\xi)
\end{aligned}
$$

Theorem 2a. The correspondence

$$
z^{k} \xi \rightarrow\left(\frac{\partial}{\partial w}\right)^{k} \xi
$$

between the elements of $L(\operatorname{sl}(2, C) ; B)$ and the B-valued differential operators of the variable $w$ introduces the structure of the LPFA in $L(\operatorname{sl}(2, C) ; B)$.

Proof. To prove the theorem we should check the following relations:

and

$$
\left[L_{0}, T(\xi)\right]=T\left(l_{0}(\xi)\right)
$$

$$
\left[L_{1}, T(\xi)\right]=T\left(l_{1}(\xi)\right)
$$

that is the easy exercise analogous to the one in Theorem 1a.

Proposition 4a. The LPFAs with the algebra of primary fields $B$ are the subfactors of $L(\operatorname{sl}(2, C) ; B)$.

The statement of the proposition is the sequence of the definition of $L(\operatorname{sl}(2, C) ; B)$.

Let's now consider the Virasoro case.

Let $B$ be an arbitrary $L^{o}$-algebra over $\left(0, C v r_{\text {reg }}\right)$. The Virasoro generators in $B$ will be denoted as $l_{j}, j=\cdots-2,-1,0,1,2, \ldots$. The operator of the left multiplication in $B$ will be denoted as $\tau$. Let's consider the left module

$$
L(C \text { vir } ; B)=L(C \text { vir }) \otimes_{0} B
$$

over the associative algebra $L(C$ vir $)$. As the linear space this module should be identified with the space of all $B$-valued holomorphic functions of the infinite set of the complex variables $c_{1}, c_{2}, c_{3}, \ldots c_{k}, \ldots$ Let's define the $C$ vir-action in 
$L(C \operatorname{vir} ; B)$ by the formulae

$$
\begin{aligned}
L_{-p}= & \sum_{k \geq 1}(k+1) c_{k+p} \frac{\partial}{\partial c_{k}}+c_{p}, \quad p>0, \\
L_{0}= & \sum_{k \geq 1} k c_{k} \frac{\partial}{\partial c_{k}}+l_{0}, \\
L_{1}= & \sum_{k \geq 1} c_{k}\left((k+2) \frac{\partial}{\partial c_{k+1}}-2 \frac{\partial^{2}}{\partial c_{1} \partial c_{k}}\right)+2 l_{0} \frac{\partial}{\partial c_{1}}+l_{1}, \\
L_{2}= & \sum_{k \geq 1} c_{k}\left((k+3) \frac{\partial}{\partial c_{k+2}}-\left(4 \frac{\partial}{\partial c_{2}}-\left(\frac{\partial}{\partial c_{1}}\right)^{2}\right) \frac{\partial}{\partial c_{k}}\right. \\
& \left.-b_{k}\left(\frac{\partial}{\partial c_{1}}, \ldots \frac{\partial}{\partial c_{k+2}}\right)\right)+\left(4 \frac{\partial}{\partial c_{2}}-\left(\frac{\partial}{\partial c_{1}}\right)^{2}\right) l_{0} \\
& \left.+3 l_{1} \frac{\partial}{\partial c_{1}}+l_{2}+0.5 c\left(\frac{\partial}{\partial c_{2}}-\frac{\partial}{\partial c_{1}}\right)^{2}\right), \\
L_{n}= & \frac{(-1)^{n}}{(n-2) !}\left(\operatorname{ad}\left(L_{1}\right)\right)^{n-2} L_{2}, \quad n>2 .
\end{aligned}
$$

Lemma 7. $L(C$ vir; $B)$ is the left module over the $L$-algebra $L(C$ vir).

Proof. One needs to verify

$$
\begin{aligned}
& {\left[L_{0}, T(w)\right]=-T(w),} \\
& {\left[L_{1}, T(w)\right]=(T(w))^{2},} \\
& {\left[L_{2}, T(w)\right]=-(T(w))^{3} ;}
\end{aligned}
$$

that is the easy exercise analogous to the one in Lemma 5.

Let now $B$ be the algebra of geometric objects and $l_{j}, j=\cdots-2,-1,0,1,2, \ldots$ are the Virasoro generators in it.

Definition 6. The algebra of geometric objects $B$ will be called admissible if

$$
\forall v \in L(C \operatorname{vir} ; B):\left((\forall k>0) L_{-k} v=L_{-k} T(w) v=0\right) \Rightarrow(v=0) .
$$

Proposition 3b. The left module $L(C$ vir; $B)$ over the $L$-algebra $L(C$ vir $)$ possesses exactly one structure of the LCFA with the algebra of primary fields $B$ if $B$ is the admissible algebra of geometric objects.

Proof. We shall present this algebra below. Its uniqueness follows from the next lemma.

Lemma 8. Let $T$ be the operator of the left multiplication in a LCFA, realised in $L(C$ vir; $B)$ then

$$
\begin{aligned}
& T(\xi) c_{1}=c_{1} \xi-l_{-1}(\xi), \\
& T(\xi) c_{2}=c_{2} \xi-l_{-2}(\xi) .
\end{aligned}
$$

Proof. The equality (26a) should be proved in a similar way as Eq. (23) from Lemma 6. To prove Eq. (26b) one should mention that

$$
L_{-k}\left(T(\xi) c_{2}-c_{2} \xi+l_{-2}(\xi)\right)=0
$$


for all $k>0$ and $\xi$ from $B$. Let's denote

$$
v=T(\xi) c_{2}-c_{2} \xi+l_{-2}(\xi),
$$

then $T(w) v=T(\tau(w) \xi) c_{2}-c_{2} \tau(w) \xi+l_{-2}(\tau(w) \xi)$. Because the algebra $B$ is admissible the element $v$ is equal to 0 .

Theorem 2b. The isomorphism between the associative algebra $U\left(L_{+}\right) \ltimes B$, where $L_{+}=\operatorname{span}\left(L_{-p}, p>0\right)$ and the module $L(C$ vir; $B)$ (both objects are considered as vector spaces), defined as

$$
U\left(L_{+}\right) \ltimes B \ni L_{-k_{1}} \ldots L_{-k_{n}} \xi \rightarrow T\left(c_{k_{1}}\right) \ldots T\left(c_{k_{n}}\right) \xi ;
$$

introduce the structure of the LCFA with the algebra of primary fields $B$ in the module $L(C$ vir; $B)$ if $B$ is the admissible algebra of geometric objects.

Proof. Let's introduce the structure of $C$ vir-module in $U\left(L_{+}\right) \ltimes B$ by the identification

$$
U\left(L_{+}\right) \ltimes B=(U(C \text { vir }) \ltimes B) / U\left(\operatorname{span}\left(L_{k}, k>0\right)\right) .
$$

It should be verified that under the isomorphism (27) this action correspond to the action (25) that can be derived from Theorem $1 \mathrm{~b}$.

Proposition 4b. The LCFAs with the algebra of primary fields $B$, which is the admissible algebra of geometric objects, are the factors of $L(C \mathrm{vir} ; B)$.

The statement of the proposition is the sequence of the definition of $L(C \mathrm{vir} ; B)$.

Acknowledgements. The author thanks M.A. Semenov-Tian-Shansky, A.A. Kirillov, A.Yu. Morosov, S.L. Shatashvili, A.M. Perelomov, A.S. Schwarz, G. Segal, A.S. Miscenko, A.A. Rosly, A.Yu. Alekseev, Yu.A. Neretin, B.L. Feigin, D.R. Lebedev, M.L. Koncevich, B.A. Khesin, V.Yu. Ovsienko and V. Fock for stimulating discussions, assistance and comments.

The author is grateful to L.D. Faddeev for his attention and encouragement.

\section{References}

1. Witten, E.: Topological quantum field theory. Commun. Math. Phys. 117, 353-386 (1988)

2. Witten, E.: Topological sigma models. Commun. Math. Phys. 118, 411-449 (1988)

3. Witten, E.: Quantum field theory and the Jones polynomial. Commun. Math. Phys. 121, 351-399 (1989)

4. Labastida, J.M.P.: Morse theory interpretation of topological quantum field theory. Commun. Math. Phys. 123, 641-658 (1989)

5. Belavin, A.A., Polyakov, A.M., Zamolodchikov, A.B.: Infinite conformal symmetry in twodimensional quantum field theory. Nucl. Phys. B 241, 333-380 (1984)

6. Cardy, J.L.: Operator content of two-dimensional conformally invariant theories. Nucl. Phys. B 270, 186-204 (1986)

7. Anderson, G., Moore, G.: Rationality in conformal field theory. Commun. Math. Phys. 117, 441-450 (1988)

8. Moore, G.,. Seiberg, N.: Classical and quantum conformal field theory. Commun. Math. Phys. 123, 177-254 (1989)

9. Friedan, D., Shenker, S.: The analytic geometry of two-dimensional conformal field theory. Nucl. Phys. B 281, 509-545 (1987)

10. Manin, Yu.I.: Critical dimensions of string theories and dualizing sheaf on the (super)curves moduli space. Funkt. anal. i ego pril. 20 (3), 88-89 (1986)

11. Beilinson, A.A., Manin, Yu.I., Schechtman, V.V.: Sheaves of Virasoro and Neveu-Schwarz algebras. Lect. Notes Math. vol. 1289, pp. 52-66. Berlin, Heidelberg, New York: Springer 1987

12. Beilinson, A.A., Schechtman, V.V.: Determinant bundles and Virasoro algebra. Commun. Math. Phys. 118, 651-701 (1988) 
13. Koncevich, M.L.: The Virasoro algebra and the Teichmüller spaces. Funkt. anal. i ego pril. 21 (2), 78-79 (1987)

14. Tukia, P.: On infinite dimensional Teichmüller spaces. Ann. Acad. Sci. Fenn. Ser. A1 Math. 3, 343-372 (1977)

15. Lehto, O.: Univalent functions and Teichmüller spaces. Berlin, Heidelberg, New York: Springer 1986

16. Kirillov, A.A.: A Kähler structure on $K$-orbits of the group of diffeomorphisms of a circle. Funkt. anal. i ego pril. 21 (2), $42-45$ (1987)

17. Kirillov, A.A., Juriev, D.V.: The Kähler geometry on the infinite dimensional homogeneous manifold $M=\operatorname{Diff}_{+}\left(S^{1}\right) / \operatorname{Rot}\left(S^{1}\right)$. Funkt. anal. i ego pril. 20 (4), 79-80 (1986)

18. Kirillov, A.A., Juriev, D.V.: The Kähler geometry on the infinite dimensional homogeneous space $M=\operatorname{Diff}_{+}\left(S^{1}\right) / \operatorname{Rot}\left(S^{1}\right)$. Funkt. anal. i ego pril. 21 (4), 35-46 (1987)

19. Kirillov, A.A., Juriev, D.V.: The highest weight representations of the Virasoro algebra by the orbit method. J. Geom. Phys., I.M. Gelfand vol. (1988)

20. Juriev, D.V.: The model of the Verma modules over the Virasoro algebra. Algebra i analiz 2 (2), 209-226 (1990)

21. Dixmier, J.: Algèbres enveloppantes. Paris: Gauthier-Villars 1974

22. Feigin, B.L., Fuchs, D.B.: Skew-symmetric differential operators on the line and the Verma modules over the Virasoro algebra. Funkt. anal. i ego pril. 16(2), 47-63 (1982)

23. Higher transcendental functions. New York: McGraw-Hill 1955

24. Gelfand, I.M., Zelevinsky, A.V., Serganova, V.V.: The general hypergeometric functions connected with the pair of the homogeneous spaces. Dokl. Akad. Nauk SSSR 304 (5), 1044-1049 (1989)

Communicated by N. Yu. Reshetikhin 
